\title{
Determination of Gestational Age: A Perspective With the Bangladeshi Ethnicity
}

\author{
M. Lutfor Rahman, PhD \\ National School of Public Health, NOVA University of Lisbon, Portugal \\ Aysha Sultana, MBBS \\ Department of Medical Ultrasound, STATE University of Bangladesh
}

Kalyan Das, PhD

Department of Basic and Applied Sciences, National Institute of Food

Technology Entrepreneurship and Management, India

Doi: 10.19044/esj.2018.v14n6p375 URL:http://dx.doi.org/10.19044/esj.2018.v14n6p375

\begin{abstract}
Determination of gestational age (GA) with precision is vital to the proper care of pregnant mothers. Our present study aimed at determining the gestational ages by the ultrasonic measurements of four standard fetal parameters namely biparietal diameter (BPD), head circumference (HC), abdominal circumference (AC), and femur length (FL) specifically focusing on the Bangladeshi ethnic population. The current study is cross-sectional but data were collected in a prospective nature. There were 229 Bangladeshi women who had usual singleton fetuses with the evidence of menstrual dates by sonography before 14 weeks. Fetal anatomical structures have been scanned and measured at the time of sonographic inspection. Multiple linear regression models of GA by the date of last menstrual period (LMP) and GA by ultrasonographic measures have been fitted on four fetal parameters for our analysis. In the present analysis, we have found that all the response variables i.e. gestational age by LMP and gestational age by ultrasonography (USG) clearly depend on the fetal parameters. The best subsets regression analysis shows that BPD, AC, and FL are the best predictors of GA by LMP $\left(\operatorname{adj} \mathrm{R}^{2}=\right.$ 96.54). In terms of Mallow's $C p$ and adj $R^{2}$, it is found that all the fetal parameters BPD, AC, HC and FL are important predictors for GA by USG. It has been observed that multiple fetal parameters measured by ultrasonography can be useful to predict gestational age during second and third trimesters. Precise estimation of gestational age by this method is helpful to reduce pregnancy-related complicacy and maternal death in the developing countries including Bangladesh.
\end{abstract}


Keywords: Fetal measurements, gestational age, ultrasonography

\section{Introduction}

Determination of gestational age (GA) in early gestation is valuable in detecting growth abnormality in later stages of pregnancy (Shehzad et al., 2006). GA can be measured by accurate record of the date of last menstrual period (LMP) or by ultrasongraphic measurements. In a developing country, similar to Bangladesh where most of the females do not keep a record of their LMP, estimation of GA of fetus by ultrasonographic fetal measurements are important for ante-preterm care and successful planning of intervention and delivery.

Generally, the calculation of pregnancy date is based on the first day of the last menstrual period (LMP) in a normal 28-day menstrual cycle with the assumption that all women can recall the date of their LMP reliably. However, even though the date of LMP can be estimated successfully, the factors, for instance, oligo-ovulation and hormone therapy might delay ovulation and thus can cause imprecise determination of pregnancy dating (Konje et al., 2002). Therefore, estimation of gestational age by other methods including ultrasound methods deserve more attention. However, Butt et al. (2014) noted that calculation of duration of a pregnancy using ultrasonographic measurements is clinically better than using last menstrual dates when ultrasonographic measurements are performed with quality and precision.

In last few decades, several researchers studied mainly four important fetal parameters namely biparietal diameter (BPD), head circumference (HC), abdominal circumference (AC), femur length (FL) separately or individually not altogether in consideration to determine GA through modeling approach. Among these four parameters BPD is one of the key parameters in determining age of the unborn baby up to 36 weeks. Kurtz et al. (1980) reveals that there is an increase in BPD with the advancement of gestational age between 17 week and term with a discrepancy of approximately 3 weeks. Jaiswal et al. (2015) assessed gestational age in second and third trimesters by ultrasonographic measurements of the fetal parameter biparietal diameter (BPD) in the southern part of Rajasthan. However, they did not use any regression model to determine GA for any given measurement of BPD. Therefore, it is not possible to determine GA accurately by their method for a given value of BPD.

Konje et al. (2002) measured gestational age by fetal kidney length measurements between the 24 and 38 weeks of gestation to distinguish its accurateness with the other fetal biometric indicators. They fitted several linear regression models with different set of fetal indices as explanatory 
variables. In their study, the predictor model of gestational age that combines biparietal diameter, fetal kidney length, head circumference, femur length and abdominal circumference appeared to be more sensible and realistic. Though they showed that their method performs better than others but it was applicable only for later stages of a pregnancy, particularly 24 weeks to 38 weeks of gestation.

Butt et al. (2014) compared performance of different fetal parameters for determination of gestational age. They recommended to use BPD or crown-rump length (CRL) for determining gestational age in the firsttrimester, in the second and third trimesters, estimation of gestational age could be done by measuring BPD, HC, AC, and FL. However, individual use of these parameters could result to vary gestational age by 7 to 35 days.

There are some shortcomings of using single fetal parameter for determining gestational age. For instance, the BPD is not consistent to assess gestational age in case of deviations in skull shape, for example, brachycephaly or dolichocephaly (Butt et al., 2014). The HC performs better as a single parameter to assess gestational age than the other three fetal parameters BPD, FL, and AC in the second trimester, and it turn out to be less precise jointly with all other parameters with the advancement of pregnancy (Konje et al., 2002). Law et al. (1982) also advocated for HC instead of BPD in the estimation of growth- adjusted sonographic age (GASA) which provides a reasonable way of assessing both fetal age and fetal growth potential in the later weeks of pregnancy.

Bensen and Doubilet (1991) noted that accuracy of all predictors of GA decreases with the advancement of pregnancy. However, among four predictors the performance of AC is significantly less than others as AC cannot be measured properly due to altering the shape of $\mathrm{AC}$ with fetal respiration and central body flexion (Butt et al. 2014; Bensen et al. 1991).

Hill et al. (1992) demonstrated that use of multiple parameters enhances the accuracy by reducing bias during gestational age estimation in second trimester. More parameters are also useful if any one gestational parameter is distorted by a fetal abnormality, such as achondroplasia on femur length (Butt et al. 2014). Therefore, to increase the precision, many researchers consider regression equations by combining several gestational parameters instead of taking account of a single fetal parameter. Although gestational age was determined by using fetal parameters individually in some studies in Bangladesh (Jaiswal et al. 2015), there is no study to determine gestational age taking into account four fetal parameters simultaneously, particularly with reference to Bangladeshi ethnic population. The studies conducted by Ashrafunnesa et al. (2003) did not estimate GA directly; they merely showed charts and graphs to present values of fetal parameters conditional on the values of GA by LMP. Given the motivation, the current study intends to 
assess gestational age in second and third trimesters by using four fetal parameters simultaneously following multiple linear regression methodology.

\section{Materials and methods}

This is a cross-sectional study, where data were collectd in a prospective nature, consisted of 229 singleton pregnant mothers enrolled during December 2015 to November 2016 in Ibn Sina Diagnostic and Imaging Center, and Ad-din Hospital in Dhaka, Bangladesh. Informed verbal consent was obtained from all participating mothers. Participants who could recall last normal menstrual period certainly, had regular menstrual cycles before pregnancy and had an ultrasonogrphic assessment before 14 weeks demonstrating a crown-rump length that matched with the time length of LMP by one week had been included in the study. Further, the patients who had the criteria of multiple gestation, maternal complications such as maternal diabetes or abnormal glucose tolerance test, pregnancy-induced hypertension, chronic hypertension, pre-eclampsia, eclampsia, placental abruption, Rh isoimmunization, drug abuse, severe oligohydramnios or polyhydramnios, abnormal fetal karyotyping, fetal congenital abnormalities have been excluded from our study.

Four fetal parameters namely biparietal diameter (BPD), head circumference (HC), abdominal circumference (AC), and femur length (FL) have been measured through ultrasonographic technology. The thalamic view has been used to measure BPD and HC. In thalamic view, it displays the thalamus, third ventricle, falx cerebri and cavum septum pellucidum (CSP) or the fornices anteriorly. Though some several methods can be used to measure BPD, but the common established method is measurement from outer-toinner. In our study, the calipers for BPD measurement have been placed at the widest distance vertical to the midline on the leading edges of the near and far parietal bones (Figure 1).

To measure the HC accurately, the elliptical measurement cursor should be placed at the outer edge of the of the skull bones, not including the skin tissue. The calipers for occipito-frontal diameter measurement have been placed on a plane perpendicular to the biparietal diameter at the midpoint of the frontal and occipital bones. The cephalic index has been calculated as the ratio of biparietal diameter to occipitofrontal diameter (Figure1). 


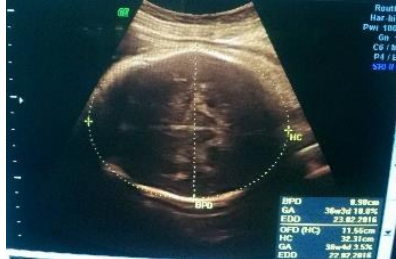

Figure1: Bi-parietal diameter (BPD) and head circumference (HC)

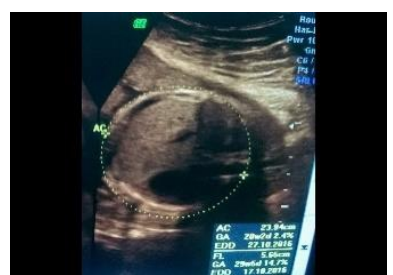

Figure 2: Abdominal circumference $(\mathrm{AC})$

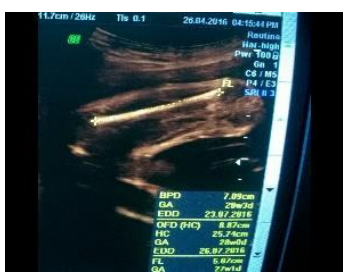

Figure 3: Femur length (FL)

Abdominal circumference (AC) is measured through a particular plane of section where fetal abdomen appeared round or nearly round. Sonographic marker for the correct AC level included the fetal spine, stomach and portal vein. The AC can predict gestational age better in the second trimester with declining precision about to term. Similarly, biological variation and risk factors may cause the factual error of AC measurements. However it should be noted that the abdominal circumference shown in Figure 2 is the gestational growth parameter that is widely affected during pregnancies affected by weird fetal growth behaviour.

The fetal femur can be measured as early as 12 weeks of gestation. The proper plane of section is the long axis of the bone when the femur is horizontal and shadows uniformly- at least from end to end. One example of measuring FL has been shown in Figure 3.

Hadlock et al. (1982) combined several measurements to increase the accuracy of gestational age assessment with the rationale that when two or more parameters estimate the same end point, the chance of predicting that end point with accuracy is improved. The BPD, HC, AC, and FL measurements are found as described before and the gestational ages corresponding to these parameters are averaged to obtain a standard gestational age. However, if gestational age measurements under various parameters are quite dissimilar, averaging several parameters might decrease the accuracy of the predictors. While certain anomalies, for example, fetal macrosomia, intrauterine growth retardation, and congenital anomalies are traced, then averaging of fetal growth parameters appears to be inappropriate (Butt et al. 2014).

In the current research, to increase accuracy of determination of gestational age, multiple linear regression models have been fitted for GA by LMP and GA by USG with BPD, FL, AC, and HC. The results have been compared by observed and predicted values of responses, adjusted R-square, Mallow's $\mathrm{Cp}$, and square root of mean squared error (S). 


\section{Results}

The main idea of analyzing data is to relate gestational age with fetal parameters. Initially a matrix plot of variables shown in Figure 4 is observed to see linear relationships of the variables. The matrix plot shows that there are clear linear relationships among each pair of explanatory variables namely BPD, $\mathrm{HC}, \mathrm{AC}$, and FL.

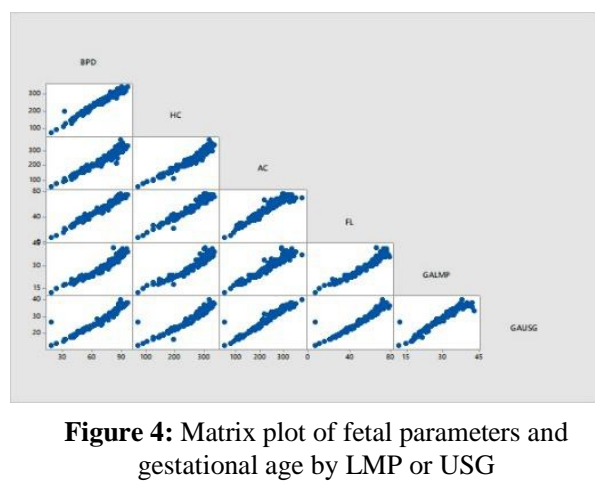

Also, it reveals that the responses (gestational age by LMP and gestational age by USG) evidently depend on BPD, HC, AC, and FL.

We use adjusted $\mathrm{R}^{2}$, Mallows' $\mathrm{C}_{\mathrm{p}}$ and mean square error to compare models with different number of predictors. Instead of simple $\mathrm{R}^{2}$ adjusted $\mathrm{R}^{2}$ has been used as $\mathrm{R}^{2}$ increases with the size of subset. $\mathrm{R}^{2}$ is most useful when comparing models of the same size. Choosing models with the highest adjusted $\mathrm{R}^{2}$ is equivalent to choosing the model with smallest mean square of the error (MSE). If the model fits the data well, the expected value of Mallows' $\mathrm{Cp}$ is approximately equal to the number of parameters in the model.

Table 1 : Best Subsets Regression of GALMP versus BPD, HC, AC, FL

\begin{tabular}{rrrrrrrrrr}
\hline Vars & R-sq & R-sq (adj) & R-sq (pred) & Mallows' $C_{p}$ & S & BPD & HC & AC & FL \\
\hline 1 & 95.5 & 95.5 & 95.4 & 72.7 & 1.4609 & & & & X \\
\hline 1 & 95.4 & 95.4 & 95.3 & 79.8 & 1.4784 & X & & & \\
2 & 96.4 & 96.4 & 96.3 & 12.5 & 1.3004 & & & X & X \\
2 & 96.3 & 96.3 & 96.2 & 21.9 & 1.3266 & X & & X & \\
3 & 96.6 & 95.5 & 96.4 & 4.5 & 1.2751 & X & & X & X \\
3 & 96.4 & 96.4 & 96.3 & 14.4 & 1.3031 & & X & X & X \\
4 & 96.6 & 96.6 & 96.4 & 5.0 & 1.2737 & X & X & X & X \\
\hline
\end{tabular}

Each line of the output in the best subset regression analysis shown in Table 1 represents a different model. The values of $\mathrm{R}^{2}$ for models tends to 100 percent which indicates all the models are explained by their independent variable(s). For GALMP the predictor model which includes BPD, AC, and FL is the best as it has the highest value of adjusted $\mathrm{R}^{2}$ (96.5), a low Mallows' $\mathrm{C}_{\mathrm{p}}$ value (4.5) and the lowest $\mathrm{S}$ value (1.2751). It is evident that the chosen model has the Mallows' $\mathrm{C}_{\mathrm{p}}$ value roughly equals the number of parameters in the model. Though inclusion of multiple explanatory variables offers benefit in model fitting, often a single parameter, for example FL, can predict the response GA reasonably (adjusted $\mathrm{R}^{2}=95.5$ ). However, as the value of Mallows' $\mathrm{C}_{\mathrm{p}}$ is very large (72.7) in case of choosing the best fitted model with the sole predictor FL, evidently it is not the best option at the end. The stepwise regression analysis shown in Table 2 confirms that BPD, AC, and FL 
are significant predictors (p-values < 0.05) for GA based on LMP. From Table 3 the regression equation for GA by LMP can be written as GALMP $=5.418$ $+0.104 \mathrm{BPD}+0.035 \mathrm{AC}+0.154 \mathrm{FL}$.

Table 2: Stepwise Regression Analysis of GALMP versus BPD, HC, AC, FL

\begin{tabular}{lrrrrr}
\hline Source & DF & Adj SS & Adj MS & F-Value & P-Value \\
\hline Regression & 3 & 10221.7 & 3407.25 & 2095.77 & 0.000 \\
BPD & 1 & 16.2 & 16.20 & 9.96 & 0.009 \\
AC & 1 & 61.5 & 61.50 & 37.83 & 0.000 \\
FL & 1 & 31.5 & 31.50 & 19.38 & 0.000 \\
Error & 222 & 360.9 & 1.63 & & \\
$\quad$ Lack-of-Fit & 179 & 360.5 & 2.01 & 193.35 & 0.000 \\
$\quad$ Pure Error & 43 & 0.4 & 0.01 & & \\
Total & 225 & 10582.7 & & & \\
\hline
\end{tabular}

Table 3: Coefficients under Stepwise Regression Analysis of GALMP

\begin{tabular}{lllrrr}
\hline Term & Coef & SE & T-Value & P-Value & VIF \\
\hline Constant & 5.418 & 0.4940 & 10.97 & 0.000 & \\
BPD & 0.104 & 0.0330 & 3.16 & 0.009 & 48.35 \\
AC & 0.035 & 0.0057 & 6.15 & 0.000 & 21.13 \\
FL & 0.154 & 0.0349 & 4.40 & 0.000 & 45.42 \\
\hline
\end{tabular}

Table 4: Best Subsets Regression of GAUSG versus BPD, HC, AC, FL

\begin{tabular}{cccccccccc}
\hline Vars & R-sq & R-sq (adj) & R-sq (pred) & Mallows' $C_{p}$ & S & BPD & HC & AC & FL \\
\hline 1 & 98.0 & 98.0 & 98.0 & 509.1 & 0.9230 & & & X & \\
\hline 1 & 97.8 & 97.8 & 97.8 & 590.1 & 0.9728 & & & & X \\
2 & 99.3 & 99.3 & 99.3 & 38.0 & 0.5495 & & & X & X \\
2 & 99.1 & 99.1 & 99.1 & 113.1 & 0.6244 & X & & X & \\
3 & 99.4 & 99.4 & 99.3 & 15.7 & 0.5242 & X & & X & X \\
3 & 99.3 & 99.3 & 99.3 & 39.0 & 0.5493 & & X & X & X \\
4 & 99.4 & 99.4 & 99.4 & 5.0 & 0.5109 & X & X & X & X \\
\hline
\end{tabular}

The best subset regression analysis of GA based on USG (see Table 4) shows that all of the fetal parameters are important for GA based on USG. This is confirmed by the stepwise regression analysis (Table 5 and Table 6) where BPD, HC, AC, and FL are significant at $5 \%$ level of significance. From Table 6 , the regression equation for GA by USG can be written as GAUSG $=$ $6.028+0.089 \mathrm{BPD}-0.012 \mathrm{HC}+0.048 \mathrm{AC}+0.146 \mathrm{FL}$. 
Table 5: Stepwise Regression Analysis of GAUSG versus BPD, HC, AC, FL

\begin{tabular}{lrrrrr}
\hline Source & DF & Adj SS & Adj MS & F-Value & P-Value \\
\hline Regression & 4 & 9638.66 & 2409.66 & 9231.09 & 0.000 \\
BPD & 1 & 9.40 & 9.40 & 36.01 & 0.000 \\
HC & 1 & 3.32 & 3.32 & 12.70 & 0.000 \\
AC & 1 & 105.57 & 105.57 & 404.41 & 0.000 \\
FL & 1 & 28.02 & 28.02 & 107.35 & 0.000 \\
Error & 221 & 57.69 & 0.26 & & \\
$\quad$ Lack-of-Fit & 178 & 57.32 & 0.32 & 37.05 & 0.000 \\
$\quad$ Pure Error & 43 & 0.37 & 0.01 & & \\
Total & 225 & 9696.35 & & & \\
\hline
\end{tabular}

Table 6: Coefficients under Stepwise Regression Analysis of GAUSG

\begin{tabular}{lrrrrl}
\hline Term & Coef & SE Coef & T-Value & P-Value & VIF \\
\hline Constant & 6.028 & 0.224 & 26.96 & 0.000 & \\
BPD & 0.089 & 0.015 & 6.00 & 0.000 & 60.46 \\
HC & -0.012 & 0.003 & -3.56 & 0.000 & 36.38 \\
AC & 0.048 & 0.002 & 20.11 & 0.000 & 23.64 \\
FL & 0.146 & 0.014 & 10.36 & 0.000 & 46.27 \\
\hline
\end{tabular}

Table 7: A random sample of data with four fetal biometry, observed and fitted gestational age by LMP (GALMP), observed and fitted gestational age by USG (GAUSG), and respective residuals

\begin{tabular}{ccccccccccc}
\hline ID & BPD & HC & AC & FL & GALMP & $\begin{array}{c}\text { Fitted } \\
\text { GALMP }\end{array}$ & $\begin{array}{c}\text { RES } \\
\text { GALMP }\end{array}$ & GAUSG & $\begin{array}{c}\text { Fitted } \\
\text { GAUSG }\end{array}$ & $\begin{array}{c}\text { RES } \\
\text { GAUSG }\end{array}$ \\
\hline $\mathbf{1 7 5 1 2}$ & 66.2 & 237.5 & 216.5 & 54.9 & 28.20 & 28.31 & -0.11 & 28.60 & 27.52 & 1.08 \\
$\mathbf{1 7 5 9 5}$ & 93.4 & 315.2 & 317.5 & 71.7 & 38.10 & 37.25 & 0.85 & 36.20 & 36.33 & -0.13 \\
$\mathbf{1 7 5 7 7}$ & 90.0 & 323.9 & 311.3 & 75.4 & 40.40 & 37.25 & 3.15 & 36.40 & 36.16 & 0.24 \\
$\mathbf{1 7 3 1 4}$ & 53.2 & 185.6 & 182.7 & 37.4 & 22.10 & 23.08 & -0.98 & 22.20 & 22.80 & -0.60 \\
$\mathbf{2 3 4 4 8}$ & 84.0 & 293.2 & 296.9 & 68.7 & 33.50 & 35.09 & -1.59 & 34.20 & 34.33 & -0.13 \\
$\mathbf{4 2 5 2 9 6}$ & 78.6 & 287.2 & 283.1 & 62.0 & 32.40 & 33.01 & -0.61 & 31.60 & 32.27 & -0.67 \\
$\mathbf{2 5 0 6 6}$ & 50.4 & 195.5 & 180.7 & 35.7 & 22.50 & 22.46 & 0.04 & 21.60 & 22.08 & -0.48 \\
$\mathbf{4 6 2 5 4}$ & 70.7 & 264.2 & 240.4 & 55.8 & 27.57 & 29.75 & -2.18 & 28.71 & 28.88 & -0.16 \\
$\mathbf{4 8 8 1 2}$ & 85.6 & 298.6 & 295.7 & 68.0 & 33.71 & 35.10 & -1.39 & 33.29 & 34.24 & -0.96 \\
$\mathbf{4 9 9 0 0}$ & 93.2 & 300.6 & 320.7 & 73.1 & 37.14 & 37.55 & -0.41 & 37.14 & 36.85 & 0.30 \\
$\mathbf{4 0 5 2 9}$ & 84.8 & 308.2 & 285.6 & 62.4 & 34.30 & 33.81 & 0.49 & 33.00 & 32.75 & 0.25 \\
$\mathbf{4 0 5 3 7}$ & 38.1 & 144.0 & 126.8 & 25.5 & 18.00 & 17.73 & 0.27 & 17.60 & 17.52 & 0.08 \\
$\mathbf{4 9 0 9 4}$ & 69.0 & 248.4 & 211.9 & 50.7 & 26.40 & 27.79 & -1.39 & 26.60 & 26.80 & -0.20 \\
$\mathbf{4 9 8 0 6}$ & 79.4 & 288.2 & 264.6 & 62.0 & 34.40 & 32.45 & 1.95 & 31.40 & 31.44 & -0.04 \\
$\mathbf{4 9 9 1 3}$ & 79.0 & 287.3 & 269.9 & 65.5 & 34.10 & 33.13 & 0.97 & 32.10 & 32.18 & -0.08 \\
\hline
\end{tabular}

There is little evidence of lack of fit in the regression analysis of GALMP and GAUSG (see Table 2 and Table 5). Perhaps this is partially due to some extreme values in the data or little misspecification of the model. Removing, for example, case numbers 5966, 57072 and 52742 from the data improves a little in the lack of fit inference, but could not eliminate the problem completely. Yet, the proposed model performs satisfactorily in predicting GA measured considering LMP (see Table 5). Table 7 presents a sample of 15 observed and predicted responses along with corresponding residuals from the complete list of data. The model has been fitted using stepwise regression method. The fitted responses under GALMP are obtained from the model: GALMP $=5.418+0.104 \mathrm{BPD}+0.035 \mathrm{AC}+0.154 \mathrm{FL}$ and 
the fitted responses under GAUSG are obtained from the model: GAUSG = $6.028+0.089$ BPD $-0.012 \mathrm{HC}+0.048 \mathrm{AC}+0.146 \mathrm{FL}$. However, the fitted and observed values and residuals have been demonstrated considering 226 cases ignoring three outliers as elimination of them entails non-violation of regression assumptions and little improvements in prediction of responses.

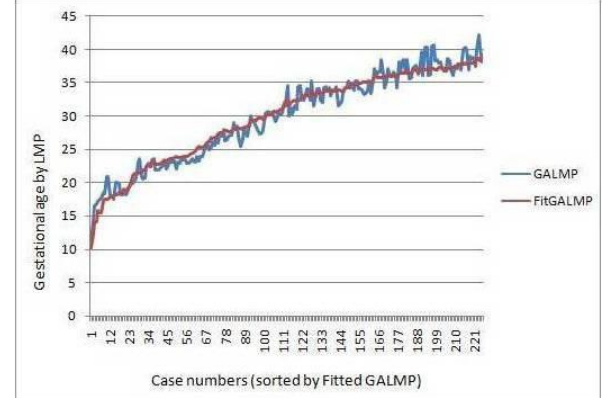

Figure 5: Observed and fitted GA by LMP Vs cases ordered by fitted LMP

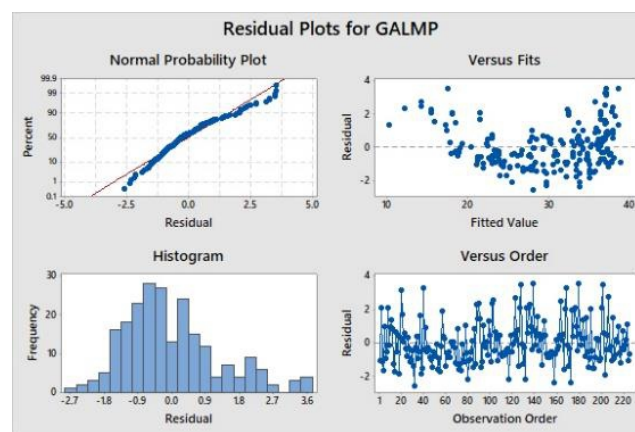

Figure 7: Residual plots for GALMP

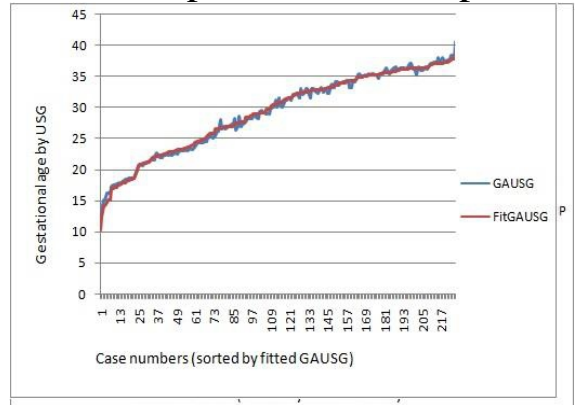

Figure 6: Observed and fitted GA by USG Vs cases ordered by fitted USG

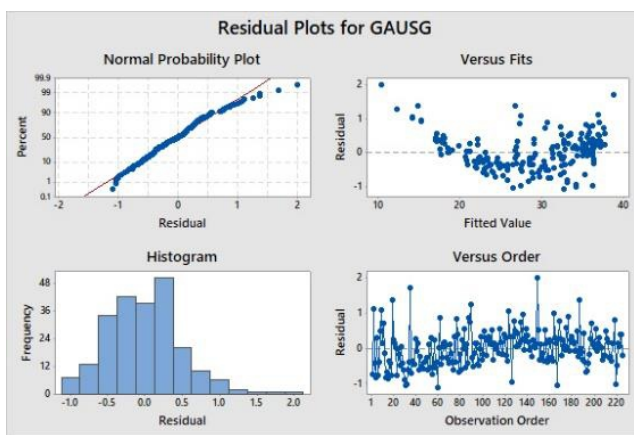

Figure 8: Residual plots for GAUSG

The Figure 5 and Figure 6 depicted observed and fitted values for GA by LMP and by USG respectively. It is found that observed and predicted values approximately overlaid each other which are also indications of good fit. However, three outliers namely case number 5966, 57072 and 52742 have been removed because of their abnormality for a smooth fit of the data. In the Figure 5 and Figure 6, x-axis denotes case numbers (ordered observed LMP or USG) and y-axis denoted gestational age.

Apparently there is no strong violation of all regression assumptions found in the residual analysis of GA under LMP (see Figure 7 and Figure 8). The residual analysis of GALMP shows that there are three outliers in the data. Excluding these three extreme cases linear models are fitted better. Throughout this study, Minitab 17 has been used in computer facilitated evaluation of fetal ultrasonic biometry. 


\section{Discussion}

Precise determination of gestational age is pivotal to quality maternity care, specifically in diagnosis of growth abnormality and timing of delivery. In this endeavour, we attempted to determine gestational age by ultrasound measurements for the population of Bangladesh.

As gestational age determination can vary with ethnicity, this study emphasizes the fact of Bangladeshi ethnicity as there has not been conducted any relevant study in Bangladesh. The current research has demonstrated the fact that multiple fetal parameters are useful criteria to predict gestational age in Bangladesh. The best subsets regression analysis shows that BPD, AC, and FL are the important predictors for gestational age calculation based on the date of last menstrual period (GALMP) as evidenced by the highest adjusted $\mathrm{R}^{2}$ (96.54), a low Mallows $\mathrm{Cp}$ (4.5) and the lowest $\mathrm{S}$ value (1.2751). The findings obtained in the best subsets regression analysis are also verified by the stepwise regression analysis which provides p- values for BPD, AC and FL much lower than $5 \%$ level of significance.

All four fetal parameters appeared to be important for the determination of gestational age by ultrasonographic measurements (GAUSG) having the highest adjusted $\mathrm{R}^{2}$ (99.39), a low Mallows Cp (5) and the lowest $\mathrm{S}$ value (0.5109) shown in the results of the best subset regression analysis (Table 4). The stepwise regression analysis also confirms that all the four predictors BPD, AC, FL and HC are significant at 5\% level for gestational age by ultrasonographic methods. Table 7 has presented a sample of observed and fitted values, residuals along with original values of fetal measurements from the full list of observed and fitted values. By reviewing this table one can reasonably presume that the prediction of gestational age by the discussant methods is sensible.

Apparently there is no strong violation of model assumptions in the analysis. However, there is a little lack of fit in the data that might be accountable to some extreme values or non-normality of the errors or little misspecification of linear models. These results could vary in different settings because of the difference in fetal parameters of different population due to ethnicity, certain conditions of pregnant mothers and some lack of fit in the data. Therefore, to generalize findings these limitations can be addressed in future studies to eliminate the shortcomings of the current study. Gestational parameters of fetus in dibetic mothers might vary due to diabetic related complexities. Therefore, fetal development in diabetic mothers could be an area of research interest in future as Bangladesh has one of the highest percentages diabetic patients in the world (Akter S. et al. 2014).

Our study reveals that multiple parameters measured by ultrasonography can be useful in gestational age determination during second and third trimesters particularly in case of unknown date of last menstrual 
period or where fundal height does not correspond with dates. Thus, precise estimation of gestational age by this method might be helpful predicting expected delivery date and reducing the pregnancy related complexity and maternal death and thereby contribute in the overall public health in developing countries like as Bangladesh.

\section{Acknowledgement}

The second author is an M.Phil researcher at the Center for Higher Studies and Research, Bangladesh University of Professionals (BUP) and acknowledges BUP for having their consent to conduct the research in this field.

\section{References:}

1. Akter S., Rahman MM., Abe SK. \& Sultana P. Bull World Health Organ 2014;92:204-213A doi: http://dx.doi.org/10.2471/BLT.13.128371

2. Ashrafunnessa, Jehan, A. U, Chowdhury, S.B, Sultana F., Uaque, J. A., Khatun S, MA Karim M.A. (2003) Construction of Fetal Charts for Biparietal Diameter, Fetal Abdominal Circumference and Femur Length in Bangladeshi Population, Bangladesh Med. Res. Counc. Bull. 2003; 29(2): 67-77.

3. Benson C, Doubilet PM. Sonographic prediction of gestational age: accuracy of second and third trimester fetal measurements. American Journal of Roentgenology. 1991;157: 1275-1277.

10.2214/ajr.157.6.1950881

4. Hadlock FP, Deter RL, Harrist RB, Park SK. Fetal biparietal diameter: A critical re-evaluation of the relation to menstrual age by means of real-time ultrasound. J Ultrasound Medicine 1982;1:97-104.

5. Hill LM, Guzick D, Hixson J, Peterson CS, Rivello DM. Composite assessment of gestational age: a comparison of institutionally derived and published regression equations. American Journal of Obstetrics and Gynecology. March 1992; 166(2):551-555. •

6. Jaiswal P, Masih WF, Jaiswal S, and Chowdhary DS. Assessment of fetal gestational age by ultrasonic measurement of bi-parietal diameter in the southern part of Rajasthan. Med J D Y Patil Uni, Jan-Feb 2015, Vol 8, Issue 1, pp. 27-30.

7. Kimberly Butt, MD, Fredericton NB Ken Lim, MD, Vancouver BC Determination of Gestational Age by Ultrasound. J Obstet Gynaecol Can 2014;36(2): 171-181.

8. Konje JC, Abrams KR, Bell SC, and Taylor DJ. Determination of gestational age after the 24th week of gestation from fetal kidney length measurements. Ultrasound Obstet Gynecol 2002; 19: 592-597. 
9. Kurtz AB, Wapner RJ, Kurtz RJ, Dershaw DD, Rubin CS, ColeBeuglet $\mathrm{C}$, et al. Analysis of biparietal diameter as an accurate indicator of gestational age. J Clin Ultrasound 1980;8:319-26.

10. Law RG, MacRae KD. Head circumference as an index of fetal age. Journal of Ultrasound in Medicine, 1982;1:281-288.

11. Shehzad K, Ali M, and Zaidi S. Fetal Biometry. Pak J Med Sci 2006, 22: 4 p. 503-508. 